ARTICLE

\title{
Unlimited sucrose consumption during adolescence generates a depressive-like phenotype in adulthood
}

Aliou B. Gueye ${ }^{1,2,6}$, Leandro F. Vendruscolo ${ }^{1,2,7}$, Camila de Ávila ${ }^{3,4,5}$, Catherine Le Moine ${ }^{1,2}$, Muriel Darnaudéry ${ }^{3,4}$ and Martine Cador $^{1,2}$

Depression is highly prevalent worldwide, but its etiology is not fully understood. An overlooked possible contributor to the epidemic of depression is feeding styles, particularly at early age when the brain is intensely changing. We have previously reported that unlimited sucrose consumption during adolescence leads to enduring changes in brain reward function. Here, we tested the hypothesis that sucrose consumption during adolescence would lead to a 'depressive-like' phenotype. Adolescent male rats were given unlimited access to $5 \%$ sucrose in their home cages from postnatal day 30 to postnatal day 46 and their emotional behavior was subsequently examined at adulthood. Sucrose consumption during adolescence caused anhedonia, decreased motivation for saccharin, increased immobility in the forced swim test and exacerbated anxiety-like behavior. Additionally, sucrose consumption during adolescence decreased cell proliferation in the hippocampus in adulthood. Chronic treatment with imipramine (10 mg/kg) normalized behavior and restored cell proliferation in the hippocampus of adult rats with a history of sucrose consumption during adolescence. A similar sucrose consumption starting at adulthood only increases immobility in the forced swim test, suggesting that sucrose intake affects also adults' behavior but to a lesser degree. Overall, our findings reveal an unsuspected protracted effect of sucrose consumption on behavior and suggest that unlimited sucrose consumption during critical periods of brain development may play an important role in the etiology of reward-related disorders such as depression.

Neuropsychopharmacology (2018) 43:2627-2635; https://doi.org/10.1038/s41386-018-0025-9

\section{INTRODUCTION}

Adolescence is a unique period for brain development [1]. A large literature indicates in both humans and rodents that the adolescent brain is particularly responsive to environmental challenges, such as stress [2,3] and drugs of abuse [4-6], which are known to promote vulnerability to psychiatric disorders. Recent evidence suggests that nutrition could also affect adolescent behavior and brain [7, 8] but very few studies have investigated the long-term effects of adolescent feeding styles on adult mental health.

The consumption of drinks rich in simple sugars (e.g., sucrose, glucose and fructose) by adolescents has dramatically increased over the last few decades [9]. Teenagers are taking a large proportion of their daily caloric intake from sugars ( $20 \%$ of total daily caloric intake); twice as much as recommended by the World Health Organization [10]. Sweetened beverages have high hedonic values and intensely activate brain reward systems involved in the regulation of emotion and motivation [11]. Importantly, sensitivity to palatable food rewards peaks during adolescence [12]. A recent epidemiological study suggests a possible link between sweet beverages consumption and the prevalence of depression [13]; however, experimental evidence is lacking.
We have previously reported in rodents that unlimited access to sucrose during adolescence, but not during adulthood, induced a marked decrease in motivation for natural rewards later on in life [14]. We also demonstrated recently that this reduction in motivation is accompanied by a deficit in the hedonic processing of sweetness in adulthood [15]. Thus, we hypothesized that intense and repeated activation of the reward system via unlimited sucrose consumption during adolescence may interfere with brain maturation processes and cause long-lasting alterations in reward-related behaviors.

Because anhedonia (i.e., inability to feel pleasure) and reduced interest for rewarding stimuli are key symptoms of major depressive disorder (DSM-V), the present study was designed to investigate the impact of chronic sucrose intake during adolescence on depressive-like phenotype and its potential reversibility by a chronic antidepressant treatment.

\section{METHODS AND MATERIALS}

Animals and sucrose exposure during adolescence

Subjects were male Wistar rats (Charles River, Lyon, France), weighing between 50 and $75 \mathrm{~g}$ (postnatal day (PND) $30 \pm 2$ ) at the beginning of sucrose exposure. Sixteen rats were used in

\footnotetext{
${ }^{1}$ Université Bordeaux, INCIA, UMR 5287, Bordeaux F-33000, France; ${ }^{2}$ CNRS, INCIA, UMR 5287, Bordeaux F-33000, France; ${ }^{3}$ INRA, Lab. Nutrition et Neurobiologie Intégrée, UMR1286, Bordeaux, France; ${ }^{4}$ Lab. Nutrition et Neurobiologie Intégrée, UMR1286, Université de Bordeaux, Bordeaux, France and ${ }^{5}$ Faculté de Médecine, Département de Psychiatrie et de Neurosciences, Centre de recherche de I'Institut universitaire de cardiologie et de pneumologie de Québec, Université Laval, Québec, QC G1V 0A6, Canada Correspondence: Martine Cador (martine.cador@u-bordeaux.fr)

${ }^{6}$ Present address: Département de pharmacologie et physiologie, Faculté de Médecine, Université de Montréal, Montréal, Canada

${ }^{7}$ Present address: Neurobiology of Addiction Section, NIDA-IRP, NIH, Baltimore, Md, USA

Aliou B.Gueye and Leandro F.Vendruscolo contributed equally to this work. Muriel Darnaudéry and Martine Cador share senior authorship.
}

Received: 7 August 2017 Revised: 10 January 2018 Accepted: 5 February 2018

Published online: 27 February 2018 
Experiment 1 and 30 rats were used in Experiment 2. The rats were individually housed and maintained in a temperature-controlled $\left(22^{\circ} \mathrm{C}\right)$ and humidity-controlled $(60 \%)$ room with an inverted 12:12 h light/dark cycle (lights on at 17:00 h). Water and food (Scientific Animal Food \& Engineering, France; containing 16.5\% protein, $59 \%$ carbohydrate and 3\% fat; with a caloric value of 2.9 $\mathrm{Kcal} / \mathrm{g}$ ) were available ad libitum throughout except during behavioral testing or otherwise stated. Experiments were conducted during the dark phase of the light/dark cycle between 09:00 and 17:00 $\mathrm{h}$.

After 4 days of habituation in the laboratory facilities, adolescent rats were given continuous free-choice between a bottle of water and a bottle of 5\% sucrose from PND 30-46 \pm 2 days. This period was selected because it is considered to approximate adolescence in humans [1]. The control group had access to two bottles of water. Fluid consumption, food intake and body weight were measured every other day. The position of the bottles was alternated every day to control for position preference. Following 16 days of sucrose consumption, all animals were given access to water in their home cages until the end of the experiments. Experiments were conducted in accordance with French (Directive 87/148, Ministère de I'Agriculture et de la Pêche) and European (Directive 2010/63/EU, 22 September 2010) legislation and approved by Institutional Regional Committee for animal experimentation (Approval $\mathrm{n}^{\circ}$. 5012088-A and $n^{\circ} 11668-A$ from the Bordeaux Ethics Committee (CNREEA $n^{\circ} 50$ ). Every effort was made to minimize suffering and the number of animals used.

Experiment 1: effects of sucrose consumption during adolescence on sucrose preference, motivation for a sweet solution and immobility in the forced swim test at adulthood. An outline of Experiment 1 is shown in Fig. 1a. Rats were exposed to water (ADO water group, $n$ $=8$ ) or sucrose (ADO sucrose group, $n=8$ ) during the adolescence period (PND30 to PND46) and were tested at adulthood (from PND60 onwards) in a battery of preclinical behavioral tests for depression: sucrose preference test, motivation for sweet solution in operant chamber and the forced swim test. Glycemia in an oral glucose tolerance test (OGTT, Supplemental Methods) was also measured to control potential metabolic alterations after adolescence unlimited sucrose consumption.

Sucrose preference test

Adult (PND70) rats with free access to food were given two bottles of water for 3 days in their home cages. Next, one bottle of water was switched to a bottle that contained $1 \%$ sucrose for $24 \mathrm{~h}$. The percentage of preference was calculated as follows: sucrose intake/total fluid intake $\times 100$. Decreased sucrose preference is classically used as a measure of anhedonia.

Motivation for a sweet solution in operant chambers

Motivation for saccharin was studied in 30 min daily sessions (5 sessions per week) in operant chambers (Imétronic, Pessac, France) equipped with two levers, as previously reported [14] (details in Supplemental Methods). Adult rats (PND85-PND160) were trained to press one of the two levers (the active lever) under a fixed-ratio 1 schedule (FR1) of reinforcement (i.e., every active lever press was reinforced by fluid delivery) to obtain $0.1 \mathrm{ml}$ of saccharin solution $(0.13 \%$, w/v; Sigma-Aldrich, Lyon, France) delivered in $3 \mathrm{~s}$. The delivery of saccharin was paired with a $4 \mathrm{~s}$ cue light located above the active lever during which additional lever presses did not result in fluid delivery (time out period). Responses on the other lever (the inactive lever) were recorded as a measure of non-specific activity. The position of the active and inactive levers was counterbalanced between rats. The rats were first tested on FR1 (16 sessions) and then shifted to a FR5 (5 lever presses resulted in fluid delivery) schedule for 8 additional sessions. The criterion for acquisition of saccharin was a minimum of 50 reinforcements per session over 2 consecutive sessions in the FR1 schedule.

After the FR5 testing, rats were tested under a progressive ratio (PR) schedule of reinforcement (5 sessions) in which the number of active lever presses required for the subsequent saccharin delivery increased progressively by 3 . The PR session was terminated when animals failed to earn a new reinforcer in 30 min or when the total session time $(120 \mathrm{~min})$ was elapsed. Data are expressed as the average breakpoint reached during 5 consecutive sessions of progressive ratio. The breakpoint was defined as the last ratio completed before the response was disrupted and was used as an index of motivation [16].

Forced swim test (FST)

Rats (PND274) were placed in a Plexiglas cylinder $(51 \mathrm{~cm}$ height, $21 \mathrm{~cm}$ diameter) filled with water $\left(24 \pm 1^{\circ} \mathrm{C}\right)$ to a level of $29 \mathrm{~cm}$ for $15 \mathrm{~min}$ during the pretest session (day 1) and $24 \mathrm{~h}$ later, for $5 \mathrm{~min}$ during the test session (day 2). Immobility was automatically quantified using a video-track FST software (View Point, Lyon, France). Immobility time was used as an index of depressive-like behavior.

Experiment 2: effects of chronic imipramine on changes associated with unlimited sucrose consumption during adolescence. An outline of the experiment 2 is shown in Fig. 2a. All analyses involving manual assessments were conducted blind of experimental group assignments (novelty-suppressed feeding test (NSFT), social interaction and $\mathrm{BrdU}^{+}$cell counting).

\section{Adolescent sucrose exposure and chronic antidepressant} treatment

Adolescent rats (sucrose group: $n=16$; water group: $n=16$ ) were given access to sucrose for 16 days from PND30 to PND46, as described in experiment 1. From PND47 (i.e., the day after the end of sucrose exposure) until the end of the experiment (PND274), animals have access only to water and $1 \mathrm{~h}$ after the lights were turned on, rats received daily intraperitoneal injections of either imipramine hydrochloride (Sigma-Aldrich, Saint-Quentin Fallavier, France) at $10 \mathrm{mg} / \mathrm{kg} / 2 \mathrm{ml}$ or vehicle (saline; $2 \mathrm{ml} / \mathrm{kg}$ ).

Motivation for a sweet solution in operant chambers Motivation for saccharin (PND65-PND116) was assessed in a progressive ratio test, as described in experiment 1 . One rat from the sucrose group treated with saline and one rat from the water group treated with imipramine did not reach the acquisition criterion for saccharine self-administration $(<50$ reinforcers after 16 sessions of FR1)and thus were excluded from the study while most of the other animals reached the criterion in 2-6 sessions.

Novelty-suppressed feeding test (NFST)

Animals (PND204) were food deprived for $24 \mathrm{~h}$ before being placed for $10 \mathrm{~min}$ in an open field $(100 \times 100 \times 30 \mathrm{~cm})$ where a pre-weighed amount of regular food was placed in the center. Latency to reach food was used as an index of anxiety-like behavior. When rats reached the food, they were returned to their homecages with food access for 5 min to test the latency to reach the food in a familiar non stressful environment.

Plasma corticosterone levels in response to restraint stress Animals (PND210) were exposed to a 30 min restraint stress in a plastic tube $(7 \mathrm{~cm}$ diameter, $19 \mathrm{~cm}$ long) and blood samples were collected 0, 30, 60 and 120 min after the stress for corticosterone determination (see details in Supplemental Methods).

\section{Social interaction}

Social behavior (PND232) was studied in a circular arena $(54 \mathrm{~cm}$ diameter, $45 \mathrm{~cm}$ high) where a pair of animals without previous common social experience (same group, same treatment, and 
A

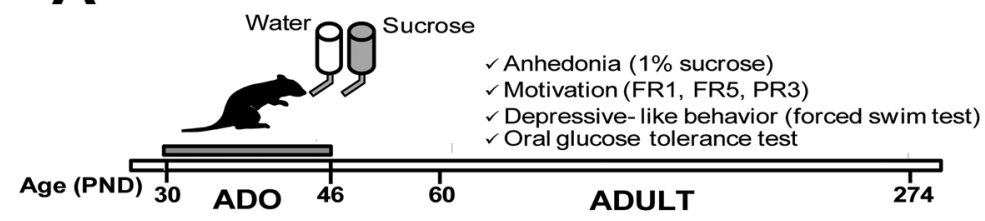

B



C

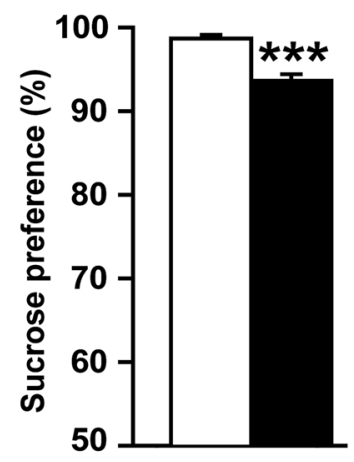

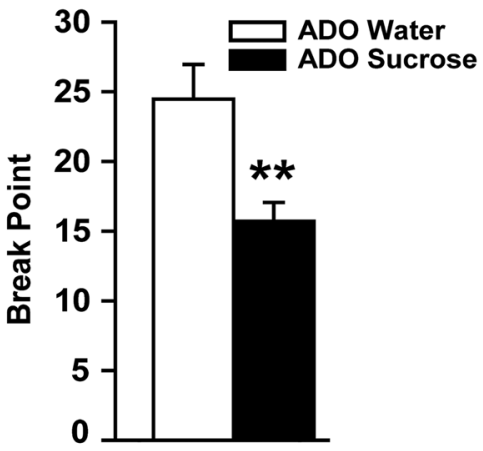

$\mathbf{E}$



Fig. 1 Unlimited sucrose consumption during adolescence promotes depressive-like phenotype at adulthood. (A) Timeline of Experiment 1. (B) Ad libitum access to $5 \%$ sucrose during adolescence strongly increased fluid intake. Rats exposed to sucrose during adolescence displayed at adulthood (C) a lower preference for $1 \%$ sucrose in a two-bottle free-choice test, (D) a decreased motivation to work for saccharinsweetened water in a progressive ratio test, and (E) an increased immobility time in the forced swim test compared with control rats. ${ }^{*} p<0.05$, ${ }^{* *} p<0.01,{ }^{* * *} p<0.001$ group exposed to sucrose during adolescence $(n=8)$ versus the group exposed to water during adolescence ( $\left.n=8\right)$. $\# p<0.05, \# \# \#<0.001$ for within comparison with age PND32 
A

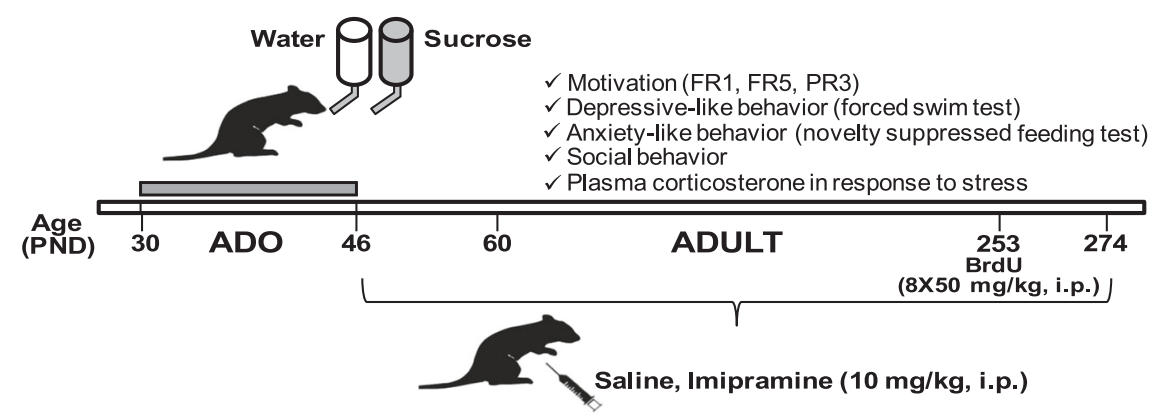

B

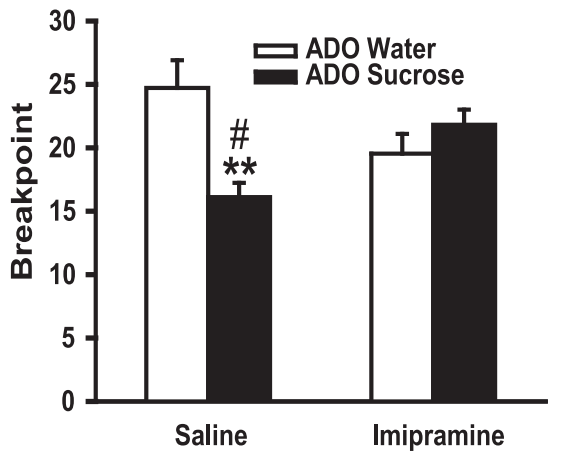

D

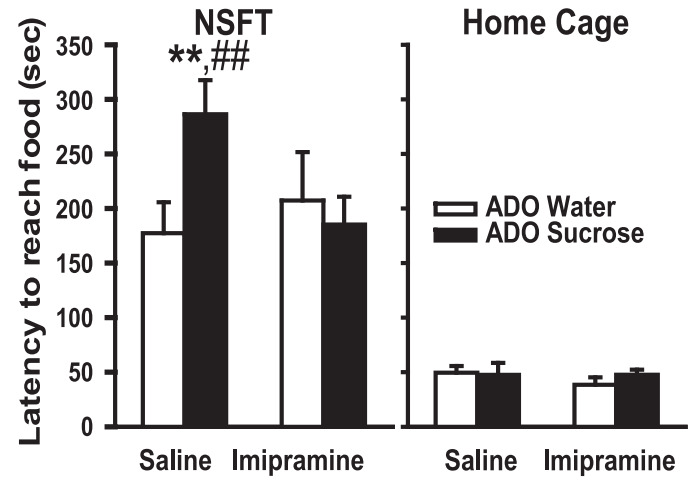

$\mathbf{F}$

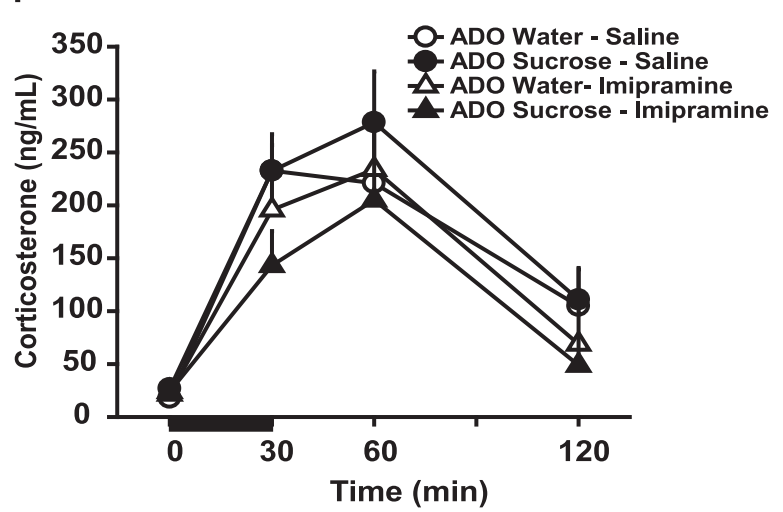

C

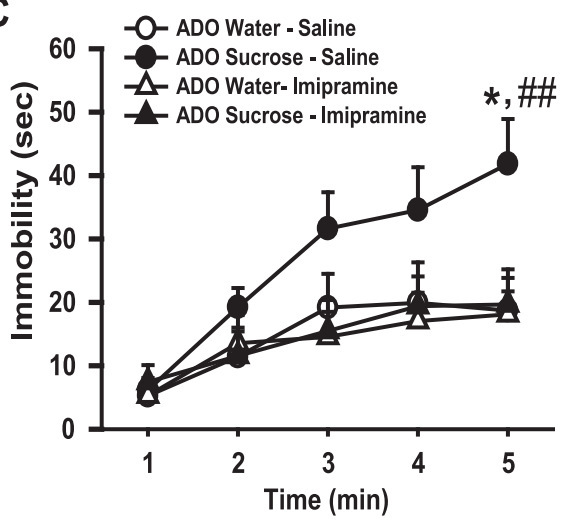

$\mathbf{E}$

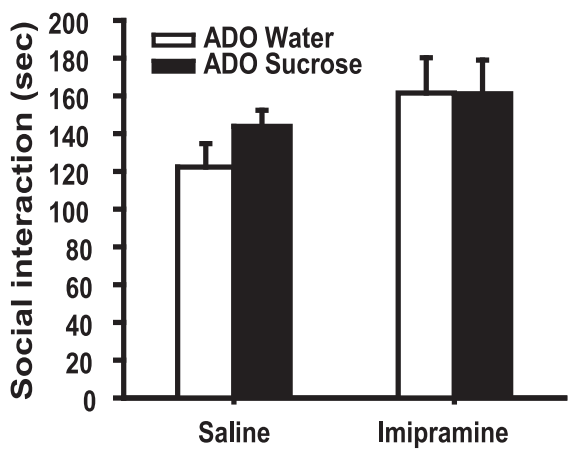

G

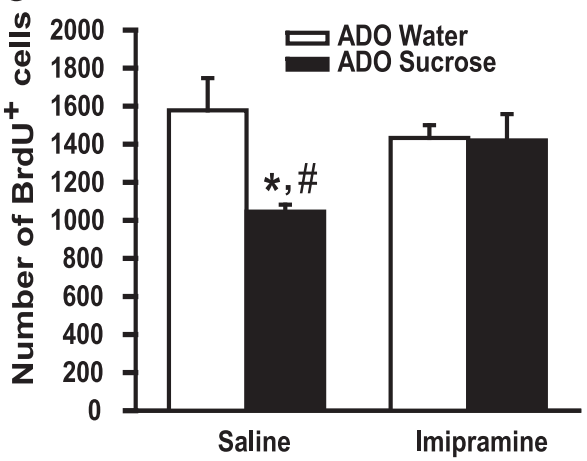

Fig. 2 Chronic antidepressant treatment reversed depressive-like phenotype produced by unlimited sucrose consumption during adolescence. (A) Timeline of Experiment 2. (B) Breakpoint for saccharin in the progressive ratio test. (C) Immobility time in the forcedswimming test. (D) Latency to reach the food in a novel environment in the NSFT (left panel) or in the homecage (right panel).(E) Time spent in social interaction between two unfamiliar conspecifics (same group, same treatment). (F) Plasma corticosterone levels before (time 0 ) and 30,60 , and 120 min post-restraint stress. (G) Number of BrdU positive cells in the dentate gyrus of the hippocampus. Newman-Keuls: ${ }^{*} p<0.05$,

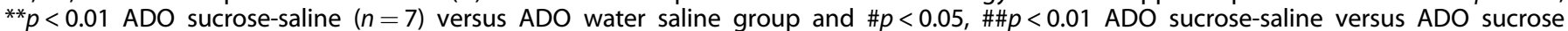
imipramine. ADO water saline group $(n=8)$, ADO-Water imipramine $(n=7)$, ADO sucrose-saline $(n=7)$, ADO-sucrose imipramine $(n=8)$ 
same body weight $\pm 10 \mathrm{~g}$ ) was placed for a $10 \mathrm{~min}$ encounter session that was video recorded (see details in Supplemental Methods).

Forced swim test (FST)

Rats (PND234-235) were tested in the FST, as described in experiment 1.

Cell proliferation in the dentate gyrus of the hippocampus Rats (PND250-253) received intraperitoneal injections of BrdU (Sigma-Aldrich, Saint-Quentin Fallavier, France), $50 \mathrm{mg} / \mathrm{kg} / 5 \mathrm{ml}$, twice daily for 4 consecutive days. Three weeks after the last BrdU injection, the rats were deeply anesthetized and transcardially perfused with phosphate buffer saline and $4 \%(\mathrm{w} / \mathrm{v})$ paraformaldehyde (PFA). Brains were harvested and post-fixed overnight in PFA at $4{ }^{\circ} \mathrm{C}$. Hippocampal cell proliferation number was assessed using BrdU immunohistochemistry [17], as described in Supplemental Methods. The counting of BrdU labeled cells along the rostro-caudal axis of the dentate gyrus (DG) was performed on coded sections with a $\times 100$ microscope lens (DM5500B, Leica). The results are expressed as the total number of cells in the whole DG (details in Supplemental Methods).

Experiment 3: effects of unlimited sucrose consumption during adulthood. Adult male Wistar rats (Charles River, Lyon, France), weighing between 260 and $270 \mathrm{~g}$ (around 90 days old), were given continuous free-choice between a bottle of water and a bottle of $5 \%$ sucrose for 16 days ( $n=8$ per group) and then maintained on water until the end of the experiment, as previously described (see experiment 1 and 2). One month after the end of the sucrose exposure, animals were tested in a battery of behavioral tests including: sucrose preference test, NSFT and the FST, as previously described (see experiment 1 and 2).

\section{Statistical analysis}

Data are expressed as means and s.e.m. The impact of sucrose exposure on behaviors was analyzed by unpaired Student's $t$-tests (for sucrose preference test and breakpoint in the operant task), or two-way analysis of variance (ANOVAs) with repeated measures (for fluid intake and immobility in the FST). To analyze the effects of imipramine, we used two-way or three-way ANOVAs (group, treatment and time). When appropriate, ANOVA was followed by Newman-Keuls post hoc tests. Social behavior data were not normally distributed and were analyzed using the non-parametric Kruskall-Wallis ANOVA. Statistical significance was defined as $p<0.05$.

\section{RESULTS}

Sucrose consumption during adolescence leads to a depressivelike phenotype at adulthood

Overall, the total fluid intake increased across days during adolescence (two-way ANOVA with repeated measures, day effect, $\mathrm{F}_{7,98}=20.68, p<0.001$; Fig. $1 \mathrm{~b}$ ), but the unlimited access to $5 \%$ sucrose drastically exacerbated fluid intake in the ADO Sucrose group (group effect, $F_{1,14}=65.24, p<0.001$ ). When given unlimited access to $5 \%$ sucrose solution, adolescent rats escalated their daily fluid intake and reached $80 \mathrm{ml}$ per day at PND40, whereas with an access limited to water, rats slightly increased their intake at PND36 and maintained their water intake around $20 \mathrm{ml}$ per day (group $\times$ day effect, $\left(F_{7,98}=4.27, p<0.001\right.$, post hoc Newman-Keuls ADO sucrose group, fluid intake from PND36 to 46 versus PND32, $p<0.001$; ADO Water group, fluid intake at PND36, $40,44,46$ versus PND32, $p<0.05)$. No group differences were found for total calories intake during the adolescence period (ADO water group: $56.2 \pm 1.1 \mathrm{Kcal} /$ day; ADO sucrose group: $58.4 \pm 1.2$ $\mathrm{Kcal} /$ day). Indeed, the sucrose-exposed animals reduced their chow intake across days (ANOVA, group $\times$ day effect, $F_{7,98}=2.53$, $p<0.05$ compensating the calories brought by the sucrose (Supplemental Figure S1). As a consequence, body weight gain during adolescence was similar across sucrose and water groups (Supplemental Figure S2).

Sucrose consumption during adolescence had long-lasting effects on behavior in adulthood. Adult male rats with a history of adolescent sucrose consumption exhibited a diminished preference for a $1 \%$ sucrose solution $\left(t_{14}=5.6, p<0.001\right.$; Fig. $\left.1 c\right)$ compared with the water group, suggesting that adolescent sucrose consumption may promote anhedonia at adulthood. To further characterize reward sensitivity, we examined the motivation to obtain a sweet solution (saccharin) in an operant paradigm. The acquisition of operant responding during FR1 was similar between ADO-water and sucrose groups (Supplemental Figure S3) indicating no motor or learning differences. When the schedule was switched to FR5, sucrose-exposed rats did not adapt as much as water-exposed rats to the increased work load and showed significantly lower number of active lever presses (Supplemental Figure S3) suggesting a decreased motivation for sweet reward. To directly evaluate their motivation, rats were switched to a progressive ratio schedule for 5 sessions. A lower breakpoint for saccharin was observed in sucrose pre-exposed animals, demonstrating a reduced motivation for saccharin $\left(t_{14}=3.1, p<0.01\right.$; Fig. 1d). To examine whether these decreases in reward sensitivity might be associated with depressive-like behavior, animals were later run in the forced-swim test (FST). Sucrose-exposed rats during their adolescence increased their immobility time throughout the 5-min test (Fig. 1e), as indicated by a significant group effect $\left(F_{1,14}=5.15, p<0.05\right)$. Together, our results suggest that unlimited consumption of sucrose during adolescence causes anhedonia, decreases motivation for a sweet reward, and exacerbates a passive coping strategy in adulthood, suggesting a depressive-like phenotype. Behavioral alterations reported in rats exposed to sucrose were not associated with impaired glucose regulation at adulthood during an oral glucose tolerance test (Supplemental Figure S4).

A chronic antidepressant treatment reverses depressive-like phenotype produced by sucrose consumption during adolescence. To further examine relevance of the alterations reported for depression, we studied in a new set of rats the impact of a chronic antidepressant treatment on the phenotype associated with adolescent sucrose exposure. We used the imipramine tricyclic antidepressant because it is known to ameliorate both anhedonia and impaired motivation [18]. We also evaluated the impact of the imipramine treatment on anxiety-like behavior, social interaction, hypothalamic-pituitary-adrenal (HPA) axis response to stress and hippocampal cell proliferation, which have been shown to be affected by depression and are sensitive to chronic antidepressant treatment [19].

First regarding motivation, ADO-water and ADO-sucrose rats, whatever their treatment (saline or imipramine) learned similarly the FR1 independently of the saline or imipramine treatment (Supplemental Figure S5). When switched to the FR5 schedule, alike in experiment 1, ADO-sucrose rats, adapted less to the increased work load (Supplemental Figure S5). More importantly, the breakpoint for saccharin in the progressive ratio test was significantly reduced in rats exposed to sucrose during adolescence and receiving the saline treatment while a chronic imipramine treatment restored motivation to the level of controls (group $\times$ treatment interaction: $F_{1,26}=11.4 p<0.01$; Post hoc Newman-Keuls, ADO-sucrose saline versus ADO-water saline, $p<0.01$; ADO-sucrose saline versus ADO-sucrose imipramine, $p<0.05$ ) (Fig. 2b). Furthermore, the similar acquisition of the operant response during FR1 between groups suggests that the effects of imipramine were not due to an improvement of cognition (Supplemental Figure S5). Imipramine effects were also independent of locomotion as indicated by a similar number of 
A

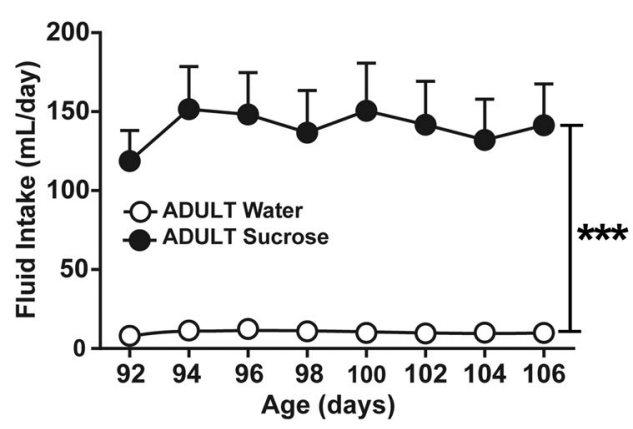

C

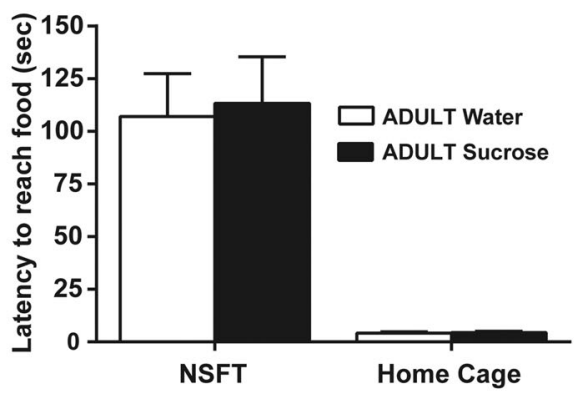

B

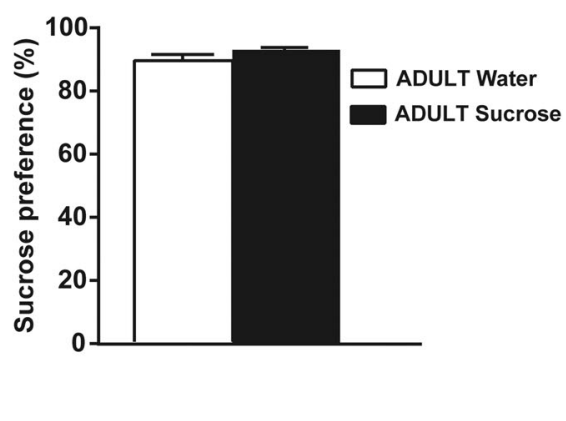

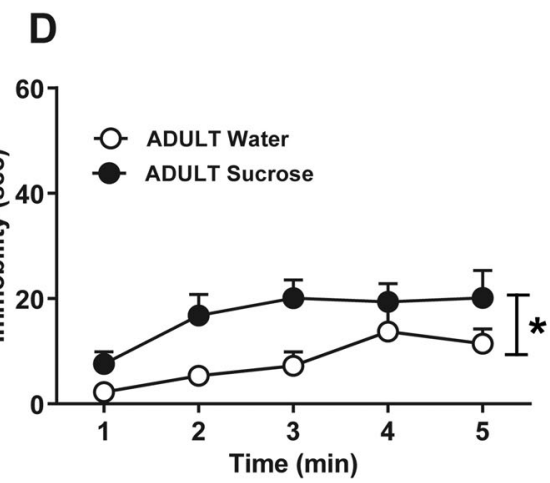

Fig. 3 Effects of sucrose consumption during adulthood on depressive-like phenotype. (A) Ad libitum access to $5 \%$ sucrose for 16 days during adulthood strongly increased fluid intake. Rats exposed to sucrose during adulthood displayed (B) no alterations in preference for $1 \%$ sucrose in a two-bottle free-choice test (C) no differences in the latency to reach food in a novel environment (NSFT, left panel) or in the home cage (right panel) and (D) an increased immobility time in the forced swim test compared with control rats. ${ }^{*} p<0.05,{ }^{* * *} p<0.001$ group exposed to sucrose during adulthood $(n=8)$ versus the group exposed to water during adulthood $(n=8)$

inactive lever presses between groups (Supplemental Figure S5). For the FST (Fig. 2c), the ANOVA revealed a significant group $\times$ treatment $\times$ time effect on immobility time $\left(F_{4,104}=2.72, p<0.05\right)$. In saline-treated groups, rats exposed to sucrose during adolescence displayed higher immobility time compared with waterexposed rats (post hoc Newman-Keuls test, minute 5, $p<0.05$ ) which replicate results of the experiment 1 . Imipramine normalized this effect by restoring the immobility scores of sucroseexposed rats to the levels of water-exposed rats (ADO-sucrose imipramine not different from $A D O$-water imipramine while different from ADO-sucrose saline at minute $5, p=0.01$ ).

Because depression is highly co-morbid with anxiety disorders and imipramine has anxiolytic effects [20], we examined the impact of imipramine in the novelty-suppressed feeding test (Fig. 2d). Rats exposed to sucrose during their adolescence and treated with saline displayed an increased latency to reach food compared to water controls, indicating a higher anxiety-like behavior in this group. This anxiogenic-like effect was corrected by the imipramine treatment (group $\times$ treatment $\times$ latency effect: $F_{1,26}=5.27, p<0.05$, post hoc Newman-Keuls tests, ADO-sucrose saline versus $A D O$-water saline, $p<0.01$; ADO-sucrose saline versus ADO-sucrose imipramine $p<0.01$; Fig. $2 d$, left panel). In contrast, latency to reach food in a familiar environment (home cage) did not significantly differ among groups and treatments (Fig. 2d, right panel).

Given that sweet beverage consumption has been associated with social behavior dysfunctions [21], we next examined the social behavior of adult animals exposed or not to sucrose during adolescence and the impact of imipramine. Animals exposed to sucrose during adolescence did not exhibit significant changes in the time spent in social interaction and imipramine did not significantly affect social behavior (Fig. 2e). Aggressive behavior (latency and duration) was not significantly affected by sucrose exposure or imipramine treatment (data not shown).

Because sucrose consumption during adolescence appears to exacerbate behavioral response to stressful situations, we examined the HPA axis response to stress. After $30 \mathrm{~min}$ of restraint stress, plasma corticosterone drastically raised (time effect $\left.F_{3,78}=57.54, p<0.001\right)$, but there were no significant differences among groups or treatments (Fig. 2f).

Depression has been associated with hippocampal atrophy and most antidepressants and environmental interventions that confer antidepressant-like behavioral effects stimulate adult hippocampal neurogenesis $[22,23]$. In accordance, adult rats with a history of sucrose consumption during their adolescence exhibited a reduced number of BrdU positive cells in the dentate gyrus (Fig. 2g; Supplemental Figure S6 for a representative image of the BrdU staining), which was attenuated by the imipramine treatment (group $\times$ treatment interaction: $F_{1,26}=4.46, p<0.05$, post hoc Newman-Keuls tests, ADO-sucrose saline vs ADO-water saline, $p<0.05$, ADO-sucrose saline vs ADO-sucrose imipramine, $p<0.05$ and ADO-sucrose imipramine vs ADO-water imipramine, n.s.).

Sucrose consumption during adulthood has no impact on sucrose preference or anxiety-like behavior but increases immobility in the FST. Finally, to determine whether the reported effects are restricted to adolescence exposure or may also be observed after adult exposure, we explored the behavior of adult rats with a history of sucrose consumption at adulthood. As previously reported [14], when given access to $5 \%$ sucrose solution, adult rats exhibited a high daily fluid intake that reached a maximum of $151.5 \mathrm{ml}$ per day in comparison with the rats consuming water, which exhibited a fluid intake around $20 \mathrm{ml}$ per day (group effect: 
$F_{1,14}=30.25, p<0.001$; Fig. 3a). As previously observed in adolescent rats, adult animals with an unlimited access to sucrose reduced their chow intake across days (group $\times$ day interaction: $F_{7,91}=2.46, p=0.0234$; data not shown). We then examined their emotional behavior at least 1 month after this period of unlimited sucrose consumption. Rats exposed to sucrose at adulthood did not show diminished spontaneous preference for a $1 \%$ sucrose solution (Fig. 3b), neither a significant change in latency to reach food in the NSFT nor in the home cage (Fig. 3c). However, unlimited sucrose consumption at adulthood produced a significant increase in the immobility in the FST (group effect: $\left.F_{1,14}=5.19, p<0.05\right)$.

\section{DISCUSSION}

The present study shows that rats given unlimited access to sucrose during the adolescence for 16 days displayed later on at adulthood alterations indicative of a depressive-like phenotype. Specifically, compared with controls, adult rats with a history of unlimited sucrose consumption exhibited: (1) decreased preference and motivation for sweet rewards; (2) increased passive coping in an inescapable situation, (3) increased anxiety-like behavior; and (4) decreased number of new born cells in the hippocampus. Importantly, these alterations were prevented by a chronic antidepressant treatment. These data demonstrate a clear protracted and deleterious impact of sucrose consumption during adolescence on adult emotional behavior. The same unlimited access to sucrose during adulthood caused much less dramatic changes in depression-/anxiety-like behaviors.

A large body of evidence demonstrates a link between obesity, metabolic disorders and anxiety and mood disorders, in adults [24-26], as well as in adolescents [27]. In preclinical models, deleterious effects on emotional behaviors [28, 29] and hippocampal neurogenesis $[30,31]$ have been reported after prolonged consumption of diets rich in fat and/or sugar. The adverse consequences are particularly remarkable when diet consumption starts during developmental periods such as in utero, infancy or adolescence [30-34] and are often associated with metabolic impairments. Herein, as previously reported in mice [35], animals consuming sucrose during adolescence did not exhibit increased body weight or hyperglycemia, suggesting that obesity or metabolic disorders are not merely involved in emotional disturbances associated with adolescence unlimited sucrose consumption. The present findings confirm and extend previous results indicating that sucrose consumption during the adolescent period leads to a reduced motivation for palatable solutions in operant paradigms [14, 35-37]. Interestingly, blunted motivation was also reported when adolescent rats were exposed to saccharin (sweet but without calories; [14]), demonstrating that the sweet taste rather than the caloric properties of sucrose appears to be the principal factor responsible for the enduring behavioral effects seen at adulthood. Notably, this effect was found to be specific to adolescence in our previous studies where no changes in motivation were found in adult rats with a history of sucrose consumption at adulthood [14]. In the present experiment, adult exposure to sucrose had no impact on sucrose preference and anxiety-like behavior but increased immobility time in the FST. Overall these findings suggest that sucrose consumption at adulthood may also have a long-lasting impact on emotional behavior, but its effect seems less dramatic than after unlimited consumption during adolescence. The effect of sucrose consumption appears to alter the rewarding/hedonic properties of sweet/palatable solutions rather than their gustatory properties. Accordingly, in a fixed-ratio procedure, a dose-response function with saccharin $(0.03$ to $1 \%)$ indicated that a history of sucrose consumption during adolescence led to a downward shift of the curve [14], suggesting a decrease in reward efficacy (or a decreased hedonic set point). Finally, using the taste reactivity test, we have recently reported reduced facial hedonic responses to sweet tastes in adult rats exposed to sucrose at adolescence [15]. Deficit in sucrose hedonic processing is classically used as a preclinical measure of depression-like behaviors and is interpreted as anhedonia, which is a core symptom for the diagnosis of major depression.

We demonstrate here that the reward deficit reported after sucrose exposure during adolescence is part of a larger preclinical profile of depression-like phenotypes including increase of passive coping strategy, hyperanxiety behavior and low hippocampal cell proliferation. Surprisingly, in these animals, the HPA response to stress was not significantly affected by adolescent sucrose exposure. Although HPA axis dysfunctions have been recurrently reported in mood and anxiety-related preclinical studies, this system appears to be predominantly affected after chronic stress or stress early in life [38]. Additionally, only a sub-group of depressed subjects exhibited HPA axis dysfunction [39]. Finally, we reported a significant decrease of new born cells in the hippocampus that is consistent with the altered neurogenesis reported in models of depression [19]. Chronic social isolation in rats constitutes a major psychosocial stress that may precipitate anxiety-like and depressive-like symptoms such as anhedonia $[40,41]$. We cannot rule out that chronic isolation may exacerbate the effect of sucrose consumption on depressive-like phenotype in our experiment. However, we recently demonstrated a similar reduced motivation in both single-housed and group-housed rats after unlimited sucrose intake suggesting that the deleterious impact of sucrose consumption on emotional behavior is not reversed by social enrichment (unpublished results). Interestingly, a recent study reveals that sucrose consumption during adolescence in group-housed animals can also exert a negative impact on cognitive functions in tasks involving prefrontal cortex and hippocampus [42]. Finally, the sensitivity to stress generated by the exposure to multiple tests seems not exacerbated by sucrose consumption during adolescence. Indeed, group differences were already detected at the first test (i.e., sucrose preference) and HPA axis response to restraint stress was unaltered in rats exposed to sucrose. A critical finding of the present study is that a chronic antidepressant treatment alleviates deficits produced by adolescent sucrose consumption. These results reinforce the idea that chronic sucrose intake during adolescence may later affect emotional behavior and brain function. Interestingly, in chronically stressed animals, chronic imipramine treatment restores altered dopaminergic response to sucrose and motivation for sucrose (breakpoint) [18]. Of note, chronic imipramine has been reported to have no impact in control rats, which is in agreement with several studies demonstrating that chronic antidepressant administration is either without effect [43] or even produced detrimental effects in control animals with no initial behavioral deficits [44].

The mechanisms underlying the enduring/remote effects of sucrose overconsumption during adolescence on emotionality are unknown. Several brain systems have been shown to regulate reward-related processes and mood related behaviors. Among them, the hippocampus may play an important role. Previous studies suggested that the hippocampus is particularly sensitive to high-fat/high-sugar diets especially when the consumption starts during developmental periods [30, 45-47]. Highly palatable food affects the structural and functional integrity of the hippocampus [48] and high sucrose consumption activates endoplasmic reticulum stress pathways [47] and modifies synaptic plasticity in the hippocampus [49]. Hippocampus regulates DA neurons located in the ventral tegmental area (VTA) [50]. VTA-nucleus accumbens (NAC) pathway may also play an important role in the effects reported in the present study. Indeed, rats exposed to sucrose during their adolescence show a blunted neuronal activation in the NAC when they drank a small amount of sweet reward at adulthood [15]. A decreased reactivity of the NAC and extracellular dopamine to rewarding events or stimuli has been 
linked to the pathophysiology and symptomatology of depression [51]. Importantly, it has been recently demonstrated in humans that a blunted development of reward-related ventral striatum activity during adolescence predicts depressive symptoms [52]. A depressive-like phenotype has been repeatedly reported following adolescence consumption of drugs that markedly stimulate DA system [4, 53-55]. Therefore, it could be hypothesized that an overstimulation of dopaminergic VTA-NAC system during adolescence via repeated consumption of sucrose might lead to enduring hyporesponsiveness of the reward system favoring the emergence of a depressive-like phenotype. Using a preclinical model of depression induced by a chronic social stress, an increased release of brain-derived neurotrophic factor (BDNF) from VTA dopamine neurons into NAC has been shown to mediate the behavioral abnormalities [56]. It has also been reported increased VTA-NAC dopamine neuron phasic firing in mice susceptible to depressive-like behavior $[57,58]$ indicating that either hyporesponsiveness or hyper-responsiveness of the reward system may be involved in the depressive-like phenotype. Because chronic high-fat and high-sugar diet consumption increases BDNF protein levels in the NAC [28], future studies should be conducted to examine the impact of the unlimited sucrose intake on BDNF levels in the NAC and its potential involvement in the detrimental effect of sucrose on emotional behavior.

In conclusion, our study provides evidence that unlimited sucrose consumption during adolescence (and adulthood to a much lesser degree), produces enduring effects at adulthood indicative of a depressive-like phenotype that could be prevented by a chronic antidepressant treatment. Overall, our work, in parallel to a growing recent literature $[7,13]$ raises the question of the long-lasting consequences of teenagers' food habits on mental health. Both sugar consumption and depression rates have dramatically increased in the last few decades in developed countries $[9,59]$ and, as suggested herein, may be causally linked. In this context, limiting sugar intake, especially in youth, may have an important impact on mental health.

\section{ACKNOWLEDGEMENTS}

The authors would like to thank Stephane Lelgouach, Anne Fayoux, Yoan Salafranque for taking care of the animals, Marion Rincel for social behavior scoring, and Marie Hélène Bruyères, Elisabeth Drillon and Marie-Laure Rousseau for administrative help.

\section{FUNDING}

This research and $A B G$ were supported by a grant to M.C. from Mission Interministérielle de la Lutte contre les Drogues et la Toxicomanie (MILDT \# 0901, Paris France). M.D. was supported by the Centre de la Recherche Scientifique (CNRS). L.F.V. was supported by Fyssen Foundation and is currently supported by NIDA-IRP. C. A. was supported by Fonds de recherche du Québec-Santé (FRQS).

\section{ADDITIONAL INFORMATION}

Supplementary information accompanies this paper at https://doi.org/10.1038/ s41386-018-0025-9 and contains supplementary material.

Competing interests: The authors declare no competing interests.

Publisher's note: Springer Nature remains neutral with regard to jurisdictional claims in published maps and institutional affiliations.

\section{REFERENCES}

1. Spear L. Modeling adolescent development and alcohol use in animals. Alcohol Res Health J Natl Inst Alcohol Abus Alcohol. 2000;24:115-23.

2. Andersen SL, Teicher MH. Stress, sensitive periods and maturational events in adolescent depression. Trends Neurosci. 2008;31:183-91.

3. Eiland $\mathrm{L}$, Romeo RD. Stress and the developing adolescent brain. Neuroscience. 2013:249:162-71.
4. Iñiguez SD, Warren BL, Parise EM, Alcantara LF, Schuh B, Maffeo ML, et al. Nicotine exposure during adolescence induces a depression-like state in adulthood. Neuropsychopharmacol Publ Am Coll Neuropsychopharmacol. 2009;34:1609-24.

5. Müller-Oehring EM, Kwon D, Nagel BJ, Sullivan EV, Chu W, Rohlfing T, et al. Influences of age, sex, and moderate alcohol drinking on the intrinsic functional architecture of adolescent brains. Cereb Cortex. 2017;7:1-15.

6. Schwarz JM, Bilbo SD. Adolescent morphine exposure affects long-term microglial function and later-life relapse liability in a model of addiction. J Neurosci J Soc Neurosci. 2013;33:961-71.

7. Khalid S, Williams CM, Reynolds SA. Is there an association between diet and depression in children and adolescents? A systematic review. $\mathrm{Br} J$ Nutr. 2016;116:2097-108.

8. Bondi CO, Taha AY, Tock JL, Totah NKB, Cheon Y, Torres GE, et al. Adolescent behavior and dopamine availability are uniquely sensitive to dietary omega-3 fatty acid deficiency. Biol Psychiatry. 2014;75:38-46.

9. Harnack L, Stang J, Story M. Soft drink consumption among US children and adolescents: nutritional consequences. J Am Diet Assoc. 1999;99:436-41.

10. Nishida C, Uauy R, Kumanyika S, Shetty P. The joint WHO/FAO expert consultation on diet, nutrition and the prevention of chronic diseases: process, product and policy implications. Public Health Nutr. 2004;7:245-50.

11. Burger KS, Stice E. Neural responsivity during soft drink intake, anticipation, and advertisement exposure in habitually consuming youth: Neural Response to Soft Drinks. Obesity. 2014;22:441-50.

12. Friemel CM, Spanagel R, Schneider M. Reward sensitivity for a palatable food reward peaks during pubertal developmental in rats. Front Behav Neurosci. 2010;4:39.

13. Knüppel A, Shipley MJ, Llewellyn $\mathrm{CH}$, Brunner EJ. Sugar intake from sweet food and beverages, common mental disorder and depression: prospective findings from the Whitehall II study. Sci Rep. 2017;7:6287.

14. Vendruscolo LF, Gueye AB, Darnaudéry M, Ahmed SH, Cador M. Sugar overconsumption during adolescence selectively alters motivation and reward function in adult rats. PLoS ONE. 2010a;5:e9296.

15. Naneix F, Darlot F, Coutureau E, Cador M. Long-lasting deficits in hedonic and nucleus accumbens reactivity to sweet rewards by sugar overconsumption during adolescence. Eur J Neurosci. 2016;43:671-80.

16. Der-Avakian A, Markou A. The neurobiology of anhedonia and other rewardrelated deficits. Trends Neurosci. 2012;35:68-77.

17. Darnaudéry M, Perez-Martin M, Del Favero F, Gomez-Roldan C, Garcia-Segura LM, Maccari S. Early motherhood in rats is associated with a modification of hippocampal function. Psychoneuroendocrinology. 2007;32:803-12.

18. Scheggi S, Pelliccia T, Ferrari A, De Montis MG, Gambarana C. Imipramine, fluoxetine and clozapine differently affected reactivity to positive and negative stimuli in a model of motivational anhedonia in rats. Neuroscience. 2015;291:189-202.

19. Miller BR, Hen R. The current state of the neurogenic theory of depression and anxiety. Curr Opin Neurobiol. 2015;30:51-58.

20. Ramirez K, Sheridan JF. Antidepressant imipramine diminishes stress-induced inflammation in the periphery and central nervous system and related anxietyand depressive- like behaviors. Brain Behav Immun. 2016;57:293-303.

21. Choi JY, Park MN, Kim CS, Lee YK, Choi EY, Chun WY, et al. Long-term consumption of sugar-sweetened beverage during the growth period promotes social aggression in adult mice with proinflammatory responses in the brain. Sci Rep. 2017;7:45693.

22. Malberg JE, Eisch AJ, Nestler EJ, Duman RS. Chronic antidepressant treatment increases neurogenesis in adult rat hippocampus. J Neurosci J Soc Neurosci. 2000:20:9104-10.

23. Sahay A, Hen R. Adult hippocampal neurogenesis in depression. Nat Neurosci. 2007;10:1110-15.

24. Castanon N, Lasselin J, Capuron L. Neuropsychiatric comorbidity in obesity: role of inflammatory processes. Front Endocrinol. 2014;5:74.

25. Roberts RE, Deleger S, Strawbridge WJ, Kaplan GA. Prospective association between obesity and depression: evidence from the Alameda County Study. Int J Obes Relat Metab Disord J Int Assoc Study Obes. 2003;27:514-21.

26. Simon GE, Von Korff M, Saunders K, Miglioretti DL, Crane PK, Belle G van, et al. Association between obesity and psychiatric disorders in the US adult population. Arch Gen Psychiatry. 2006;63:824-30.

27. Quek YH, Tam WWS, Zhang MWB, Ho RCM. Exploring the association between childhood and adolescent obesity and depression: a meta-analysis. Obes Rev J Int Assoc Study Obes. 2017;18:742-54.

28. Sharma S, Fulton S. Diet-induced obesity promotes depressive-like behaviour that is associated with neural adaptations in brain reward circuitry. Int J Obes. 2013;37:382.

29. Zemdegs J, Quesseveur G, Jarriault D, Pénicaud L, Fioramonti X, Guiard BP. Highfat diet-induced metabolic disorders impairs $5-\mathrm{HT}$ function and anxiety-like 
behavior in mice: Correlation between metabolic disorders and anxiety. $\mathrm{Br} \mathrm{J}$ Pharmacol. 2016;173:2095-110.

30. Lépinay AL, Larrieu T, Joffre C, Acar N, Gárate I, Castanon N, et al. Perinatal highfat diet increases hippocampal vulnerability to the adverse effects of subsequent high-fat feeding. Psychoneuroendocrinology. 2015;53:82-93.

31. Boitard C, Etchamendy N, Sauvant J, Aubert A, Tronel S, Marighetto A, et al Juvenile, but not adult exposure to high-fat diet impairs relational memory and hippocampal neurogenesis in mice. Hippocampus. 2012;22:2095-2100.

32. Harrell CS, Burgado J, Kelly SD, Johnson ZP, Neigh GN. High-fructose diet during periadolescent development increases depressive-like behavior and remodels the hypothalamic transcriptome in male rats. Psychoneuroendocrinology. 2015;62:252-64.

33. Maniam J, Antoniadis CP, Youngson NA, Sinha JK, Morris MJ. Sugar consumption produces effects similar to early life stress exposure on hippocampal markers of neurogenesis and stress response. Front Mol Neurosci. 2015;8:86.

34. Sasaki A, Vega W de, Sivanathan S, St-Cyr S, McGowan PO. Maternal high-fat diet alters anxiety behavior and glucocorticoid signaling in adolescent offspring. Neuroscience. 2014;272:92-101.

35. Frazier CRM, Mason P, Zhuang X, Beeler JA. Sucrose exposure in early life alters adult motivation and weight gain. PLoS ONE. 2008;3:e3221.

36. Reichelt AC, Abbott KN, Westbrook RF, Morris MJ. Differential motivational profiles following adolescent sucrose access in male and female rats. Physiol Behav. 2016;157:13-19.

37. Vendruscolo LF, Gueye AB, Vendruscolo JCM, Clemens KJ, Mormède $P$, Darnaudéry $M$, et al. Reduced alcohol drinking in adult rats exposed to sucrose during adolescence. Neuropharmacology. 2010b;59:388-94.

38. Franklin TB, Saab BJ, Mansuy IM. Neural mechanisms of stress resilience and vulnerability. Neuron. 2012;75:747-61.

39. Zorn JV, Schür RR, Boks MP, Kahn RS, Joëls M, Vinkers CH. Cortisol stress reactivity across psychiatric disorders: A systematic review and meta-analysis. Psychoneuroendocrinology. 2017;77:25-36.

40. Fone KCF, Porkess MV. Behavioural and neurochemical effects of post-weaning social isolation in rodents-relevance to developmental neuropsychiatric disorders. Neurosci Biobehav Rev. 2008;32:1087-102.

41. Murínová J, Hlaváčová N, Chmelová M, Riečanský I. The evidence for altered BDNF expression in the brain of rats reared or housed in social isolation: a systematic review. Front Behav Neurosci. 2017;11:101.

42. Reichelt AC, Killcross S, Hambly LD, Morris MJ, Westbrook RF. Impact of adolescent sucrose access on cognitive control, recognition memory, and parvalbumin immunoreactivity. Learn Mem. 2015;22:215-24.

43. Marrocco J, Reynaert M-L, Gatta E, Gabriel C, Mocaer E, Di Prisco S, et al. The effects of antidepressant treatment in prenatally stressed rats support the glutamatergic hypothesis of stress-related disorders. J Neurosci. 2014;34:2015-24.

44. Baek IS, Park JY, Han PL. Chronic antidepressant treatment in normal mice induces anxiety and impairs stress-coping ability. Exp Neurobiol. 2015;24:156.

45. Hsu TM, Konanur VR, Taing L, Usui R, Kayser BD, Goran Ml, et al. Effects of sucrose and high fructose corn syrup consumption on spatial memory function and hippocampal neuroinflammation in adolescent rats. Hippocampus. 2015;25:227-39.

46. Janthakhin $Y$, Rincel M, Costa A-M, Darnaudéry M, Ferreira G. Maternal high-fat diet leads to hippocampal and amygdala dendritic remodeling in adult male offspring. Psychoneuroendocrinology. 2017;83:49-57.

47. Pinto BAS, Melo TM, Flister KFT, França LM, Kajihara D, Tanaka LY, et al. Early and sustained exposure to high-sucrose diet triggers hippocampal ER stress in young rats. Metab Brain Dis. 2016;31:917-27.

48. Kanoski SE, Davidson TL. Western Diet Consumption and Cognitive Impairment: Links to Hippocampal Dysfunction and Obesity. Physiol Behav. 2011;103:59.

49. Lemos C, Rial D, Gonçalves FQ, Pires J, Silva HB, Matheus FC, et al. High sucrose consumption induces memory impairment in rats associated with electrophysiological modifications but not with metabolic changes in the hippocampus. Neuroscience. 2016;315:196-205.

50. Loureiro M, Renard J, Zunder J, Laviolette SR. Hippocampal cannabinoid transmission modulates dopamine neuron activity: impact on rewarding memory formation and social interaction. Neuropsychopharmacol Publ Am Coll Neuropsychopharmacol. 2015;40:1436-47.

51. Russo SJ, Nestler EJ. The brain reward circuitry in mood disorders. Nat Rev Neurosci. 2013;14:609-25.

52. Hanson JL, Hariri AR, Williamson DE. Blunted ventral striatum development in adolescence reflects emotional neglect and predicts depressive symptoms. Biol Psychiatry. 2015;78:598-605.

53. Bolaños CA, Barrot M, Berton O, Wallace-Black D, Nestler EJ. Methylphenidate treatment during pre- and periadolescence alters behavioral responses to emotional stimuli at adulthood. Biol Psychiatry. 2003;54:1317-29.

54. Bolaños CA, Willey MD, Maffeo ML, Powers KD, Kinka DW, Grausam KB, et al. Antidepressant treatment can normalize adult behavioral deficits induced by early-life exposure to methylphenidate. Biol Psychiatry. 2008;63:309-16.

55. Briones TL, Woods J. Chronic binge-like alcohol consumption in adolescence causes depression-like symptoms possibly mediated by the effects of BDNF on neurogenesis. Neuroscience. 2013;254:324-34.

56. Berton O, McClung CA, Dileone RJ, Krishnan V, Renthal W, Russo SJ, et al. Essential role of BDNF in the mesolimbic dopamine pathway in social defeat stress. Science. 2006;311:864-68.

57. Chaudhury D, Walsh JJ, Friedman AK, Juarez B, Ku SM, Koo JW, et al. Rapid regulation of depression-related behaviours by control of midbrain dopamine neurons. Nature. 2012;493:532-6.

58. Wook Koo J, Labonté B, Engmann O, Calipari ES, Juarez B, Lorsch Z, et al. Essential role of mesolimbic brain-derived neurotrophic factor in chronic social stressinduced depressive behaviors. Biol Psychiatry. 2016;80:469-78.

59. GBD 2015 Disease and Injury Incidence and Prevalence Collaborators. Global, regional, and national incidence, prevalence, and years lived with disability for 310 diseases and injuries, 1990-2015: a systematic analysis for the Global Burden of Disease Study 2015. Lancet Lond Engl. 2016;388:1545-602. 